\title{
Highest-quality surface passivation of low-resistivity $p$-type silicon using stoichiometric PECVD silicon nitride
}

\author{
Jan Schmidt and Mark Kerr \\ Australian National University, Centre for Sustainable Energy Systems, \\ Faculty of Engineering and Information Technology, Canberra ACT 0200, Australia \\ Tel: (+61) 26279 8295, Fax: (+61) 26249 0506, E-Mail: Jan.Schmidt@ faceng.anu.edu.au
}

\begin{abstract}
The surface passivation properties of silicon nitride $(\mathrm{SiN})$ films fabricated by high-frequency direct plasma-enhanced chemical vapour deposition (PECVD) on low-resistivity $(1 \Omega \mathrm{cm}) p$-type silicon solar cell substrates have been investigated. The process gases used were ammonia and a mixture of silane and nitrogen. In order to find the optimum set of SiN deposition parameters, a large number of carrier lifetime test structures were prepared under different deposition conditions. The optimised deposition parameters resulted in outstandingly low surface recombination velocities (SRVs) below $10 \mathrm{~cm} / \mathrm{s}$. Interestingly, we find the lowest SRVs for stoichiometric SiN films, as indicated by a refractive index of 1.9. In former studies similarly low SRVs had only been obtained for very silicon-rich SiN films. The fundamentally different passivation behaviour of our SiN films is attributed to the addition of nitrogen to the process gases.
\end{abstract}

Keywords: Silicon nitride; Surface passivation; PECVD; Silicon; Solar cells

\section{Introduction}

In recent years, the low-temperature $\left(\leq 400^{\circ} \mathrm{C}\right)$ surface passivation of crystalline silicon solar cells by means of PECVD silicon nitride films has attracted an increasing attention of the PV community. These films have proven to be capable of combining an outstanding surface passivation quality with excellent antireflection properties [1,2]. On typical $p$-type silicon solar cell substrates it has been shown that, provided the ion bombardment of the silicon surface during the plasma deposition is avoided, the surface passivation quality of very silicon-rich SiN films is superior to that of state-of-the-art high-temperature silicon dioxide [3-6]. These SiN films are very well suited for the rear surface passivation of bifacial solar cells, as has been demonstrated with efficiencies exceeding 20\% [7]. However, the fact that these SiN films are extremely silicon rich brings about several problems: (i) the etch rates of the films are extremely low, hindering the local opening of the SiN by means of photolithography and chemical etching, (ii) the films show a considerable absorption in the UV range of the sun spectrum, leading to a reduction of the short-circuit current, and (iii) the films are very poor insulators and cannot be used at point-contacted rears of solar cells. These problems strongly limit the applicability of silicon-rich SiN films. In this work, we demonstrate for the first time that stoichiometric $\mathrm{SiN}$ (i.e., a-SiN ${ }_{x}: \mathrm{H}$ with $x \approx 1.3$ ) is capable of producing the same low $\mathrm{SRV}$ on $p$-type silicon wafers as silicon-rich $\mathrm{SiN}$, provided that nitrogen is added to the process gases of silane and ammonia during the SiN deposition. These well-passivating stoichiometric SiN films are easily etchable, do not absorb UV photons with wavelengths above $320 \mathrm{~nm}$ and act as perfect insulators.

\section{Experimental details}

The carrier lifetime test structures used to investigate the surface passivation behaviour of the SiN films were fabricated on $400 \mu \mathrm{m}$ thick, polished $1-\Omega \mathrm{cm}$-type float-zone (FZ) silicon wafers with a (100) surface orientation. Silicon nitride films with a thickness of $\sim 60 \mathrm{~nm}$ were symmetrically deposited onto both surfaces 
of the RCA-cleaned wafers. This structure has been proven to give the optimum sensitivity of the measured effective lifetime with regard to variations in the SRV [3]. The SiN films were produced in a commercially available parallel-plate reactor (Oxford Plasma Technology, Plasmalab 80+) using ammonia and a silane/nitrogen mixture (4.5\% silane, $95.5 \%$ nitrogen) as process gases. In contrast to pure silane gas, the silane/nitrogen mixture is much safer as it is not flammable in direct contact with air. To avoid the ion bombardment of the silicon surface during the plasma deposition, a high excitation frequency $(13.56 \mathrm{MHz})$ well above the plasma frequency was used. The effective carrier lifetime $\tau_{\text {eff }}$ of the samples was measured using a contactless, inductively coupled photoconductance tester (Sinton Consulting, WCT100), allowing transient photoconductance decay (PCD) as well as quasi-steady-state photoconductance (QSSPC) measurements [8]. The wavelength-dependent refractive index $n$ and extinction coefficient $k$ of the SiN films were calculated from the reflectance data recorded with a Filmetrics F20 spectrometer. In addition, some films were also investigated by means of spectral ellipsometry. The $n(\lambda), k(\lambda)$ dependencies measured by ellipsometry were found to be in good agreement with the results of the reflectance measurements.

\section{Optimisation of the PECVD parameters}

The PECVD parameters varied in this study were the substrate temperature, the total gas pressure, the plasma power, the gas flows and the silane/nitrogen-to-ammonia gas flow ratio $\left[\mathrm{SiH}_{4}: \mathrm{N}_{2}\right] /\left[\mathrm{NH}_{3}\right]$. Figure 1 shows the impact of the two most important parameters: the deposition temperature and the gas flow ratio. Also included in Fig. 1 is the refractive index $n$ of some $\mathrm{SiN}$ films at a wavelength of $630 \mathrm{~nm}$, as determined from reflectance measurements. $\tau_{\text {eff }}$ was measured by the PCD method. The $\mathrm{NH}_{3}$ flow was fixed at $50 \mathrm{sccm}$, the pressure at 0.2 Torr and the plasma power at $100 \mathrm{~W}$ during this experiment. While the plasma power had no recognisable impact on the surface passivation quality, an increase of $\tau_{\text {eff }}$ with decreasing pressure was found. Hence, we used the lowest adjustable pressure (0.2 Torr) in Fig. 1. It is important to note that the same dependence of $\tau_{\text {eff }}$ on the gas flow ratio as shown in Fig. 1 was also found for an $\mathrm{NH}_{3}$ flow of 100 sccm, indicating that the important SiN deposition parameter is indeed the ratio of the gas flows and not the actual gas flows. As can be seen from Fig. 1, there exists an optimal gas flow ratio, which lies between 4 and 7 , depending on the deposition temperature. The optimum gas flow ratio as well as the maximum $\tau_{\text {eff }}$ increase with the deposition temperature. For the highest deposition temperature used in this study $\left(400^{\circ} \mathrm{C}\right)$, a

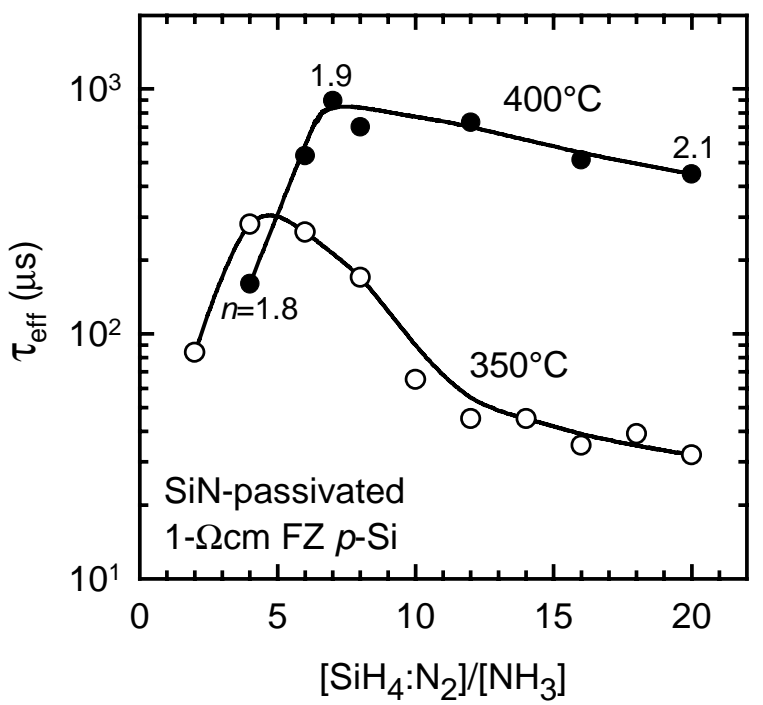

Fig. 1. Impact of the SiN deposition temperature and the silane/nitrogen-to-ammonia gas flow ratio on the effective carrier lifetime $\tau_{\text {eff. }}$. For some samples the refractive index $n$ of the SiN film is indicated. 
$\tau_{\text {eff }}$ value of $900 \mu$ s was obtained for the optimum gas flow ratio. This corresponds to a very low SRV $<10$ $\mathrm{cm} / \mathrm{s}$. Hence, the surface passivation quality of the SiN films optimised in this work is equivalent to the best $\mathrm{SiN}$ passivation scheme reported to date [3-6]. The main difference of our optimised SiN films over those investigated in Refs. 3-6 is that they are close to stoichiometry (indicated by a refractive index of 1.9) rather than very rich in silicon (refractive index > 2.2). The fundamentally different passivation behaviour of our $\mathrm{SiN}$ films seems to be due to the addition of nitrogen to the process gases. It is important to note that the silicon-rich SiN film of Fig. 1 with a refractive index of 2.1 produces a relatively good surface passivation as well. The $450 \mu$ s effective lifetime of the corresponding sample implies a sufficiently high degree of surface passivation (SRV $30 \mathrm{~cm} / \mathrm{s}$ ) for most solar cell applications. It should be noted that the same optimisation experiment has also been performed on $0.3-\Omega \mathrm{cm} \mathrm{FZ} p$-Si, giving the same set of optimal deposition parameters

\section{Injection-level dependence of the effective surface recombination velocity}

Figure 2 shows the effective SRV $S_{\text {eff }}$ as a function of the bulk injection level $\Delta n$ for a SiN-passivated sample in comparison with a thermally oxidised $1-\Omega \mathrm{cm} p$-Si wafer. The SiN deposition was performed using the optimised set of PECVD parameters. The silicon dioxide was grown at $1100^{\circ} \mathrm{C}$ in an oxygen/TCA ambient and subsequently annealed in forming gas $\left(5 \% \mathrm{H}_{2}, 95 \% \mathrm{Ar}\right)$ at $400^{\circ} \mathrm{C}$ for $30 \mathrm{~min}$ to improve the passivation quality. The $S_{\text {eff }}$ values were determined from the effective lifetimes measured with the QSSPC technique $[8,9]$ using a realistic bulk lifetime of $1.6 \mathrm{~ms}$. In order to account for the error due to the uncertainty in the assumed bulk lifetime, we have also calculated the uncertainty range of $S_{\text {eff }}$, shown as error bars in Fig. 2 [10]. In the case of the oxidised sample, the error bars were smaller than the symbol size and have therefore been omitted. As can be seen from Fig. 2, the $S_{\text {eff }}(\Delta n)$ dependence of the silicon surface passivated by the optimised $\mathrm{SiN}$ is much weaker than that of the oxidised sample. While the $\mathrm{SRV}$ at the $\mathrm{Si} / \mathrm{SiO}_{2}$ interface increases from a minimum value of $28 \mathrm{~cm} / \mathrm{s}$ at an injection level of $5 \times 10^{15} \mathrm{~cm}^{-3}$ to an $S_{\text {eff }}$ of about $700 \mathrm{~cm} / \mathrm{s}$ at $\Delta n=10^{13} \mathrm{~cm}^{-3}$, the SRV at the Si/SiN interface increases from a minimum value of $9 \mathrm{~cm} / \mathrm{s} \mathrm{at} 1.5 \times 10^{15} \mathrm{~cm}^{-3}$ to only $40 \mathrm{~cm} / \mathrm{s}$ at $10^{13} \mathrm{~cm}^{-3}$. The weak $S_{\text {eff }}(\Delta n)$ dependence of the $\mathrm{Si} / \mathrm{SiN}$ interface observed in this work is also quite different from that reported for silicon surfaces coated by highest-quality silicon-rich SiN, where the SRV increases by a factor of 25 as the injection level falls from $10^{15}$ to $10^{13} \mathrm{~cm}^{-3}[3,6]$. The lower SRV at low $\Delta n$ provided by our SiN films is very important for solar cell operation at maximum power point conditions, which typically imply $\Delta n$ values between $10^{13}$ and $10^{14} \mathrm{~cm}^{-3}$ at the rear surface of the cell.

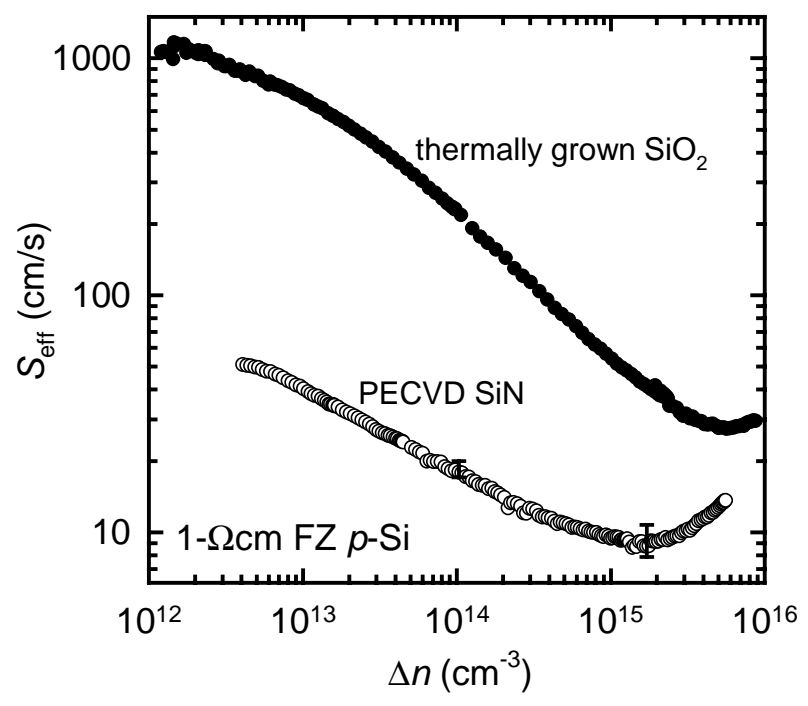

Fig. 2. Measured effective surface recombination velocity $S_{\text {eff }}$ as a function of the injection level $\Delta n$ of a SiNpassivated and a thermally oxidised and forming gas annealed low-resistivity $p$-Si wafer. 


\section{Optical properties}

The absorption coefficient $\alpha$ of the SiN films was calculated from the measured extinction coefficient $k$ using the equation $\alpha=4 \pi k / \lambda$. Figure 3 shows the wavelength dependence of the absorption coefficient for an optimised stoichiometric SiN film and a silicon-rich SiN film with refractive indices of 1.9 and 2.1 at $\lambda=$ $630 \mathrm{~nm}$, respectively. As can be seen from Fig. 3, the silicon-rich SiN film shows a pronounced absorption in the ultraviolet range of the sun spectrum, whereas the optimised stoichiometric SiN film does not absorb any UV photons with wavelengths above $320 \mathrm{~nm}$. The reduced absorption of the well-passivating stoichiometric SiN films improves the solar cell short-circuit current density by $0.5-1 \mathrm{~mA} / \mathrm{cm}^{2}$ at one sun (AM1.5G spectrum) in comparison with silicon-rich SiN films as investigated in Refs. 3-6. Moreover, stoichiometric silicon nitride has the optimum refractive index (1.9) for a single-layer antireflection coating (ARC) on silicon. However, it is important to note that the latter advantage of the stoichiometric over the silicon-rich SiN can only be exploited in laboratory solar cells, as it does not apply to encapsulated cells. In the case of glassencapsulated silicon solar cells, the optimum refractive index of a non-absorbing ARC on silicon is 2.3 and the non-optimal refractive index of stoichiometric SiN leads to a short-circuit current loss of $\sim 1.3 \mathrm{~mA} / \mathrm{cm}^{2}$, slightly overcompensating the gain from the reduced absorption. As the surface passivation quality provided by the Si-rich SiN film shown in Fig. 3 is relatively good as well, our silicon nitride with 2.1 refractive index is better suited as surface-passivating ARC for encapsulated solar cells.

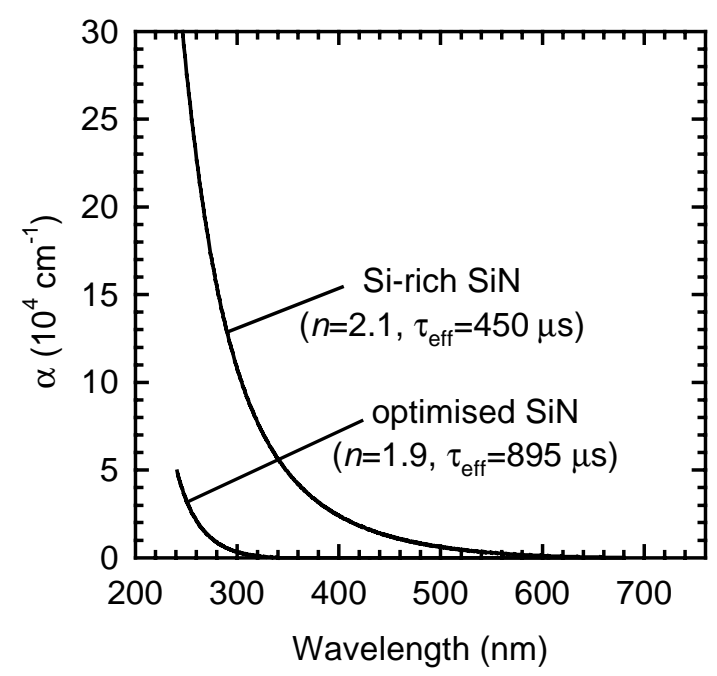

Fig. 3. Measured absorption coefficient $\alpha$ of a stoichiometric and a silicon-rich $\mathrm{SiN}$ film. The effective carrier lifetime provided by the films on $1-\Omega \mathrm{cm} p$-Si wafers and the refractive index of the $\mathrm{SiN}$ at $\lambda=630 \mathrm{~nm}$ are also indicated.

\section{Etch rates}

The etch rate of the optimised SiN in buffered HF $\left[50 \mathrm{ml} \mathrm{HF}(50 \%)+500 \mathrm{ml} \mathrm{NH}_{4} \mathrm{~F}(50 \%)\right]$ was determined to be $\sim 35 \mathrm{~nm} / \mathrm{min}$. This relatively high etch rate (the etch rate of Si-rich $\mathrm{SiN}$ with $n \geq 2.1$ was determined to be a factor of $\sim 6$ lower) allows an easy patterning of the SiN by means of photolithography and chemical etching, as has been demonstrated in our lab.

\section{Thermal stability}

A common problem of the surface passivation by means of PECVD SiN films is the instability of the passivation quality during annealing, a phenomenon which is usually attributed to the thermally activated loss of hydrogen [4,5]. In order to investigate the thermal stability of the SRV at the Si/SiN interfaces fabricated in this study, annealing experiments in the temperature range between 400 and $600^{\circ} \mathrm{C}$ in forming gas as well as 

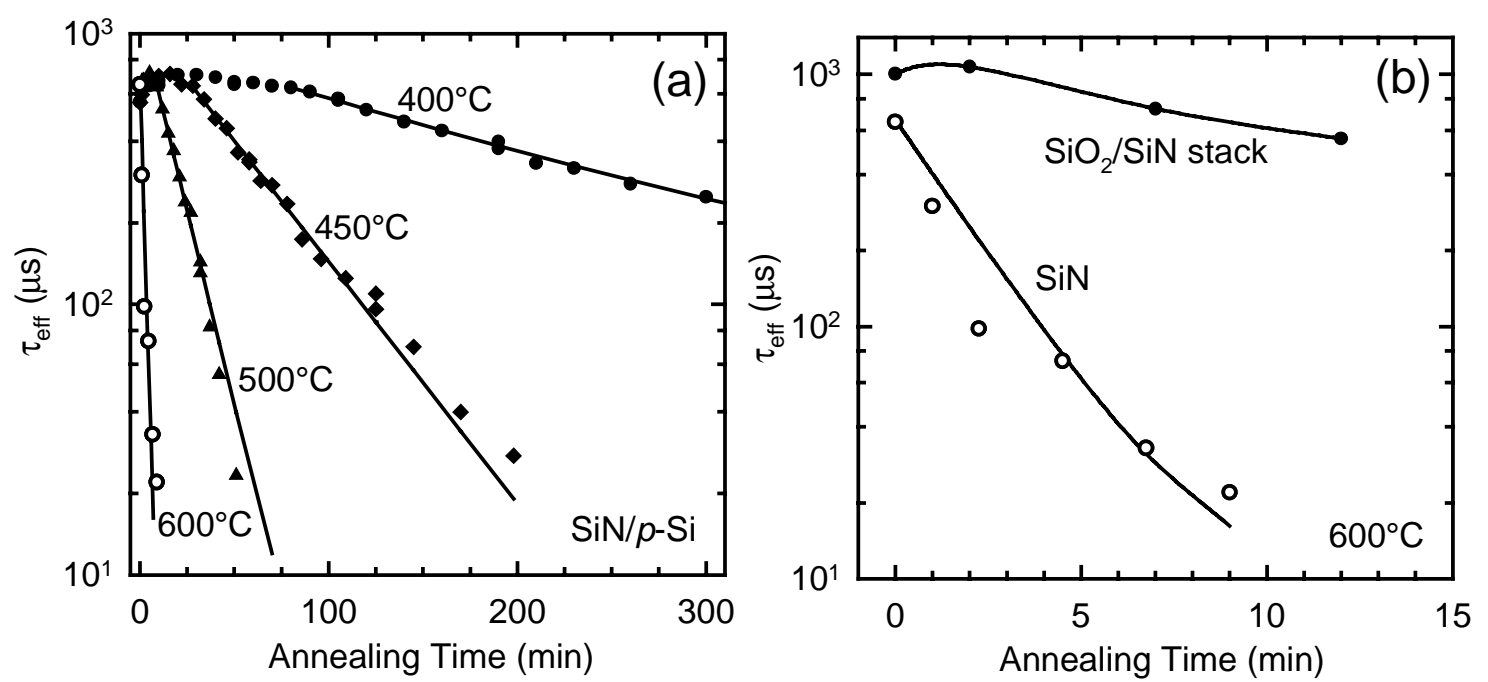

Fig. 4. (a) Measured effective carrier lifetime $\tau_{\text {eff }}$ of SiN-passivated $1-\Omega \mathrm{cm} p$-Si wafers annealed at different temperatures in nitrogen as a function of the annealing time. (b) Dependence of $\tau_{\text {eff }}$ on the annealing time $\left(600^{\circ} \mathrm{C}\right.$, nitrogen) for $1-\Omega \mathrm{cm} p$-Si wafers passivated by single-layer $\mathrm{SiN}$ films and $\mathrm{SiO}_{2} / \mathrm{SiN}$ stacks.

nitrogen were performed. No difference between the annealing behaviour of the samples in forming gas and nitrogen was observed. Figure $4 \mathrm{a}$ shows the measured effective lifetime of SiN-passivated $1-\Omega \mathrm{cm} p$-Si wafers as a function of the annealing time at different annealing temperatures. At $400^{\circ} \mathrm{C}$ the lifetime improves slightly during the first $30 \mathrm{~min}$ and for longer annealing times $\tau_{\text {eff }}$ decreases approximately exponentially. For higher annealing temperatures the degradation of $\tau_{\text {eff }}$ starts earlier and the degradation time constant decreases strongly with temperature. The solid lines in Fig. 4a show exponential fits to the measured data. From the Arrhenius plot of the degradation time constant an activation energy of $1.3 \mathrm{eV}$ was determined, in good agreement with the results reported in the literature for Si-rich films [4,5]. The increase of $\tau_{\text {eff }}$ during the first few minutes of the annealing seems to be due to the addition of nitrogen to the process gases. This phenomenon has not been observed on silicon wafers passivated by PECVD SiN films fabricated without the addition of nitrogen to the process gases [4,5]. We conjecture that it is caused by the breaking of N-H bonds and the subsequent passivation of silicon dangling bonds by the additional free hydrogen atoms.

In order to investigate if the introduction of a thin oxide layer between the silicon and the SiN is capable of improving the thermal stability of the SRV, we have investigated stacks of a very thin thermally grown $\mathrm{SiO}_{2}$ and PECVD SiN. The oxidation was performed at $900^{\circ} \mathrm{C}$ for $5 \mathrm{~min}$ in an oxygen/TCA ambient and the resulting thickness of the silicon dioxide was determined by a Filmetrics F20 spectrometer to be $8 \mathrm{~nm}$. On top of the oxidised silicon surfaces, $60 \mathrm{~nm}$ thick SiN films were deposited using the optimised parameter set of Section 3. In agreement with experimental results of Rohatgi et al. [11] obtained on very similar test structures, these oxide/nitride stacks provide an excellent degree of surface passivation, as indicated by measured $\tau_{\text {eff }}$ values around $1 \mathrm{~ms}$ (see Fig. 4b). However, even more important is the strongly improved thermal stability of the surface passivation by the oxide/nitride stacks compared to the single-layer SiN passivation. This makes the stack passivation scheme particularly well suited for solar cell fabrication processes, where the surface passivation has to withstand a higher temperature (e.g., during the firing of screen-printed contacts $[11,12])$.

\section{Conclusions}

In conclusion, we have shown that stoichiometric PECVD SiN films can be used to attain SRVs below 10 $\mathrm{cm} / \mathrm{s}$ on low-resistivity $p$-Si surfaces, provided that (i) a PECVD technique which avoids the ion bombard- 
ment of the silicon surface during the plasma deposition is used and (ii) nitrogen is added to the process gases of silane and ammonia. In addition to the outstanding surface passivation by the SiN films optimised in this study, the films provide a very weak injection level dependence of the effective SRV, they are excellent insulators and have also very beneficial optical properties: they do not absorb UV light with wavelengths above $320 \mathrm{~nm}$ and act as an effective single-layer antireflection coating on silicon. Furthermore, etching experiments with buffered HF have shown that the optimised silicon nitride can be easily patterned by means of photolithography and chemical etching. Hence, the SiN passivation scheme introduced in this work is ideally suited for point-contacted rear surfaces of high-efficiency silicon solar cells. This is in particular interesting for high-efficiency multicrystalline silicon solar cells, where a high-temperature oxidation step can severely degrade the bulk carrier lifetime of the substrate material. In addition, the large hydrogen content of the SiN films might be exploited to improve the bulk lifetime of the multicrystalline base substrate.

A second very promising field of application for the developed SiN passivation scheme are bifacial and back-contact solar cells. These types of cells require that the SiN passivation is stable under UV illumination, which has been proven for the silicon-rich SiN passivation of Refs. 3-6, but has still to be verified for the SiN passivation scheme presented in this work. A comprehensive investigation of the SiN passivation behaviour under UV illumination is currently under way in our lab.

Finally, we have demonstrated a possibility to improve the thermal stability of the SiN surface passivation by implementing a thin thermally grown silicon dioxide film between the silicon surface and the SiN film. The oxide/nitride stacks result in an excellent surface passivation with strongly enhanced thermal stability compared to the single-layer SiN passivation. Hence, the oxide/nitride double structure is ideally suited for solar cell processes where the surface passivation has to withstand a higher temperature.

\section{Acknowledgements}

The authors would like to thank C. Jagadish and H. Tan for making it possible to use the PECVD reactor at the Electronic Materials Engineering Department of the ANU. Thanks also to A. Blakers and A. Cuevas for their continuous support. One of the authors (J.S.) gratefully acknowledges the support of a Feodor Lynen Fellowship provided by the German Alexander von Humboldt Foundation. Additional funding was provided by the Australian Research Council.

\section{References}

[1] A.G. Aberle and R. Hezel, Progress in Photovoltaics 5 (1997) 29-50.

[2] C. Leguijt, P. Lölgen, J.A. Eikelboom, A.W. Weeber, F.M. Schuurmans, W.C. Sinke, P.F.A. Alkemade, P.M. Sarro, C.H.M. Maree, and L.A. Verhoef, Sol. Energy Mater. Sol. Cells 40 (1996) 297-345.

[3] J. Schmidt, T. Lauinger, A.G. Aberle, and R. Hezel, Proc. 25th IEEE Photovoltaic Specialists Conf., Washington, 1996 (IEEE, New York, 1996) 413-416.

[4] T. Lauinger, A.G. Aberle, and R. Hezel, Proc. 14th European Photovoltaic Solar Energy Conf., Barcelona, 1997 (Stephens, Bedford, 1997) 853-856.

[5] T. Lauinger, J. Moschner, A.G. Aberle, and R. Hezel, J. Vac. Sci. Technol. A 16 (1998) 530-543.

[6] J. Schmidt and A.G. Aberle, J. Appl. Phys. 85 (1999) 3626-3633.

[7] A. Hübner, A.G. Aberle, and R. Hezel, Proc. 14th European Photovoltaic Solar Energy Conf., Barcelona, 1997 (Stephens, Bedford, 1997) 92-95.

[8] R.A. Sinton and A. Cuevas, Appl. Phys. Lett. 69 (1996) 2510-2512.

[9] H. Nagel, C. Berge, and A.G. Aberle, submitted to J. Appl. Phys.

[10] J. Schmidt and A.G. Aberle, J. Appl. Phys. 81 (1997) 6186-6199.

[11] A. Rohatgi, S. Narasimha, and D.S. Ruby, Proc. 2nd World Conf. Photovoltaic Solar Energy Conv., Vienna, 1998 (EC, Ispra, 1999) 1566-1569.

[12] K. Shirasawa, K. Fukui, K. Okada, Y. Inomata, H. Takahashi, Y. Fukawa, and S. Fujii, Proc. 14th European Photovoltaic Solar Energy Conf., Barcelona, 1997 (Stephens, Bedford, 1997) 384-387. 